\title{
Mobile Application Based Modified Screening and Assessment Tools for Children with Autism
}

\author{
https://doi.org/10.3991/ijim.v13i08.10563 \\ Arpita Mazumdar ( $\left({ }^{-}\right)$ \\ Haldia Institute of Technology, Haldia, India \\ arpitamazumdar.hitegmail.com \\ Biswajoy Chatterjee \\ University of Engineering \& Management, Kolkata, India \\ Mallika Banerjee \\ Indian Institute of Information Technology, Kolkata, India \\ Irfan H Bhati \\ Haldia Institute of Technology, Haldia, India
}

\begin{abstract}
The researchers have focused on mobile application of screening tool to profile a child according to the degree of autism as per Indian Scale for Assessment of Autism (ISAA) with some modification. The work also emphasizes on practical implementation of various educational assessment tools viz. Indian Portage guide, BASIC-MR (Behavioural Assessment Scales for Indian Children with Mental Retardation) Part A, (FACP) Functional Assessment Checklist for Programming to assess children with autism in form of mobile application. The aim of this study is to enhance easiness, to improve accuracy, to monitor the child's progress and to make the whole process a time efficient one.
\end{abstract}

Keywords - ISAA, FACP, Indian Portage guide, BASIC MR (Part A), assessment tools, mobile application, ASD

\section{Introduction}

In an era where 'inclusive development' is being emphasized as the right path towards sustainable development (Disabled-persons-in-India-2016) [1] as every student can learn just not on the same day or in the same way, focused initiatives for the welfare of persons with disability are essential.

In the disability spectrum about $1 \%$ of the global population suffers from Autism Spectrum Disorder (ASD). 1 in 68 Indian is affected with this neuro developmental disorder that onsets at an early age \& limits the child's interaction with the world. It is extremely essential to diagnose or measure autism at the earliest. Childhood Autism Rating Scale (CARS) [2] is the commonly used scale for rating the severity of autism for the children more than two years of age. The scale lacks the information about the 
disability percentage. NIMH (National Institute for Mentally Handicapped) developed the Indian Scale for Assessment of Autism (ISAA) for diagnosis and measuring the severity of autism in 2009 [3].

As an assistive tool TOBY (tobyplaypad.com) a play pad application is developed to teach children with ASD by a group of scientists in Deakin University, Curtin University, Australia $[7,8]$. It acts as a technical support to an early intervention program for children with ASD between diagnosis and commencement of formal therapy.

Children with ASD exhibit individual learning abilities and disabilities. Hence educational assessment is very useful to identify their strength and weakness. Some commonly practiced standard educational tools applied on Indian population are Indian Portage guide developed by CBR network, Behavioural assessment scales for Indian Children with mental retardation (Basic-MR) (Part A/B), Functional Assessment Checklist for Programming (FACP) etc.

Our initiative adds easiness and simplicity to the process to classify a child according to the degree of autism as per Indian Scale for Assessment of Autism (ISAA) with some modification. This work also helps educator to visualize and analyze periodical assessments which may help them to design Individualized Program (IEP).

\section{About the Tools}

\section{$2.1 \quad$ ISAA}

ISAA is a screening tool for persons with Autism. Experts closely observe the person, clinically evaluate the behaviour, test by interacting the subject and seek information from parents and care givers to diagnose autism. The assessment period is about 20-30 minutes.

ISAA consists of 40 activities rated on a 5-point rating Likert scale ranging from 1(never) to 5 (always). The 40 items of ISAA are categorised under six domains viz.

1. Social relationship \& reciprocity

2. Emotional responsiveness

3. Speech language \& Communication

4. Behaviour Patterns

5. Sensory Aspects

6. Cognitive Component.

All qualitative data are further quantified for providing percentages to indicate the frequency, degree \& intensity of behavioural characteristics that are observed. The categories depending up on the occurrence of the activity observed are associated with numeric score.

- Rarely (up to $20 \%$ ) Score 1

- Sometimes $(21-40 \%)$ Score 2

- Frequently (41-60\%) Score 3

- Mostly (61-80\%) Score 4 
- Always (81-100\%) Score 5

ISAA reports the levels of autism and disability percentage of a person. ISAA is a standard psychometric tool for making diagnosis of persons with autism and is reliable and valid in Indian population.

\subsection{Indian Portage Guide to Early Childhood Education}

Portage is an international program on holistic child development. It provides knowledge on understanding the current development and plan early intervention making optimum use of potential in every child. Portage guide developed by CBR Network (South Asia) is very useful for professionals and parents invoked in the care and development of 0-6 age group children with or without special needs.

Children with autism in India often are assessed and evaluated by Portage guide developed by CBR network, India [4]. The guide considers cognitive development, language and communication, motor development, self-help skills and social development area of a child and determine the child's ability level in various academic areas. This phase is necessary before a child receives special education and related services.

Each functional area covers numerous age-related activities. The activities are rated on a 6-point rating scale (0-5) based on the levels of responses.

\subsection{Behavioural Assessment Scales for Indian Children with Mental Retardation (BASIC MR)}

The Behavioural Assessment scales for Indian children with mental retardation has been developed by National Institute for the Mentally Handicapped (NIMH) [5] in 1992 to extract systematic information about the current level of behaviour of preschoolers or school going children (3 to 16 years) with mental disorder. BASIC MR has been designed in 2 parts. - Part A and Part B.

Part A: The items included in Part A of the scale helps to assess the current level of skill behaviours in the child. The part A consists of 280 items grouped under the following seven domains viz.

1. Motor

2. Activities Daily Living (ADL)

3. Language

4. Reading-Writing

5. Number Time

6. Domestic-Social

7. Pre-Vocational-Money

We have implemented only Part A in our application as the part appropriately assess the functional areas of a child with autism during early intervention process.

Scoring of BASIC-MR (Part A): Each child with mental blockage may show different levels of performance on every item in part A. There are six possible levels of 
performance under which each item can be scored. The appropriate score obtained by the child are converted into domain wise percentage and cumulative percentage. And finally, the progress profile is plotted on graph. The Assessment is done initially and before starting the teaching program of a child with autism called baseline assessment. During teaching quarterly assessments are done. At the end of teaching program evaluation is done using this tool.

\subsection{Functional Assessment Checklist for Programming (FACP)}

The Department of Special Education, National Institute for Mentally Handicapped, Secunderabad, introduced a series of Checklists to facilitate programme planning in each child with mental handicap. [6]

There are positive checklists in this series. Each checklist considers different levels of child's functioning namely pre-primary (3-6 years of age), primary I (7-8 years of age), primary II (8-14 years), secondary group (11-14 years), prevocational-I \& II (1518 years)

The checklist covers the following areas: - Personal, Social, Academic, Occupational, Recreational. The checklist is activity based rather than skill based as the activities require multiple skills. Moreover, an activity is easier to observe and measure the progress in the given area. In addition, this tool is flexible regarding the inclusion or deletion of activities in any given area.

Scoring of FACP: Activities performed by occasional cuing or independently are counted as a point while calculating percentages. Achievement of $80 \%$ of items in the checklist is considered for promotion of the next higher level. Recreational activities are evaluated by grade system.

The tool follows the evaluation scheme followed in mainstream education system i.e., the child's progress is periodically monitored, quantified and promotion or detention is decided logically.

\section{Design of the Proposed Application}

Our goal is simple: to help special educators, parents during the stressful time of diagnosis \& commencement of formal therapy. In this work, we have implemented ISAA tool in form of mobile app for classifying a child according to the degree of severity of autism. Each item of ISAA is assessed and rated based on intensity, duration and frequency of the characteristics. In our application a special educator must input the levels of each activities based on keen observation and information supplemented by parents or care givers. ISAA tool reports the degree of autism of a child and the percentage of disability as per the score. We added a new feature i.e., domain wise degree of impairment. This may be beneficial to identify the strength and weakness of the child in a particular domain. This information may act as a guideline during baseline assessment done prior to a formal intervention program.

The standard practice in the special education setup surveyed is as follows: 
1. When child with autism comes to join a special school, he/she is keenly observed by the educators.

2. For some days (3-7days). Student's cognitive development, social development etc. are assessed.

3. Various standard educational assessment tools are consulted viz. Indian Portage guide developed by CBR network, Behavioural assessment scales for Indian Children with mental retardation (Basic-MR), FACP (Functional Assessment Checklist for Programming) (Part A/B) etc.

4. Based on the assessment, Individualized Educational Plan (IEP) is designed by the educator for each student.

5. Student is assisted with appropriate teaching learning material.

6. Periodic evaluation of their progress is done \& revised IEP is designed.

It is also aimed to represent the performance data in intelligible way using summarisation, visualisation \& interactive interfaces which will help educators /caregivers to visualize and analyze the ongoing activities of the students. In this work, we have also implemented few frequently practiced educational assessment tools [4] [5] [6] in form of mobile app. We have adapted few changes regarding scoring calculation. The modifications are due to maintain uniformity among the tools, to measure the progress of a child and to quantify the qualitative data. The educator has to observe the child's behaviour and activities and record their performance level. The performance data are to be analyzed to generate the learning pattern at regular interval. All activities irrespective of tools are rated on a 6-point rating scale (0-5). Table 1 describes the levels of responses and score assigned in each level in detail

Table 1. Levels of responses and score assigned in each level in our proposed application

\begin{tabular}{|l|l|c|}
\hline \multicolumn{1}{|c|}{ Levels } & \multicolumn{1}{c|}{ Meaning } & Score Assigned \\
\hline Total Dependent & One must do the task for the child & 0 \\
\hline Physical prompt & Child needs physical help to do the task & 1 \\
\hline Verbal prompt & Child requires verbal commands to perform the task & 2 \\
\hline Gestural prompt & Child requires gestural help to accomplish the task & 3 \\
\hline Occasional cuing & Child needs clues for thinking to perform the given task & 4 \\
\hline Independent & Child can do the task on his own & 5 \\
\hline
\end{tabular}

Domain wise percentage indicates the levels of the performance, their strength and weakness too. The report incorporates graphical representation mentioning the comparison with previous assessment done (if any). Figure 1 and Figure 2 illustrates the input screen and report graph screen of the application implementing BASIC-MR assessment tool respectively. 


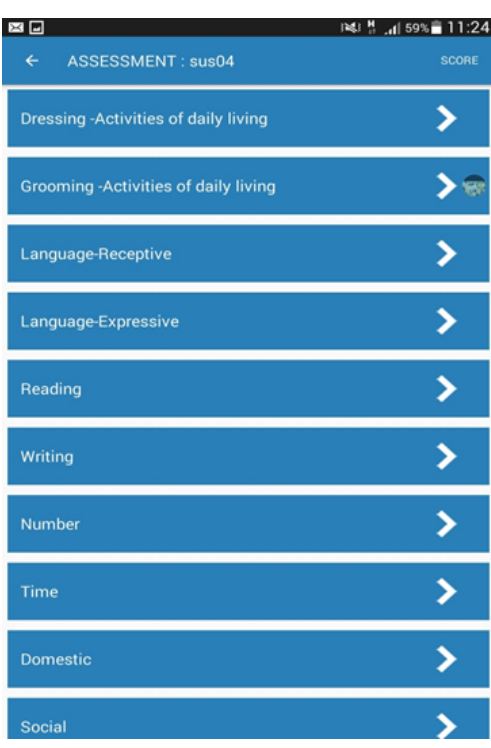

Fig. 1. Input Screen -BASIC-MR

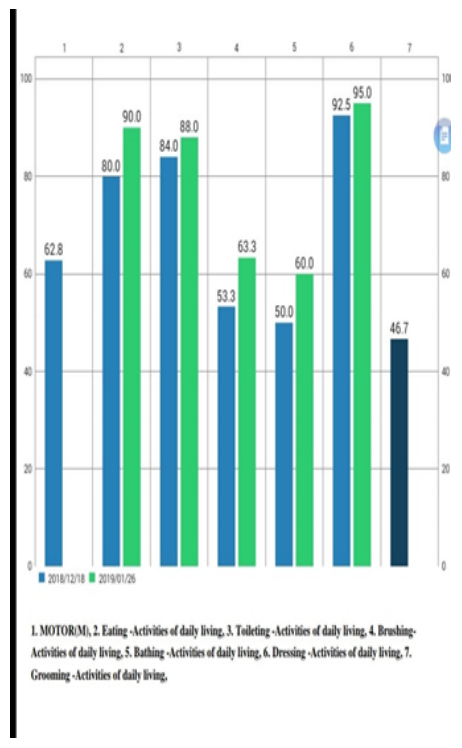

Fig. 2. Output Graph BASIC-MR

\section{$4 \quad$ Experiments}

We have undertaken 2 trials during the development of Autism Management tool app.

Trial 1: - About 20 children have been diagnosed by ISAA tool using the app. Also 10 students were assessed by Portage Guide, Basic MR (Part A), FACP (Pre-Primary) through the app. The qualitative feedback received lead to the design iterations.

Trial 2: - Trial was conducted at home of children. Trained parents of 16 children were involved in using ISAA app. Report generated by app (Figure 3 and Figure 4) is appreciated by parents as they were able to find out strength and weakness of their child in a systematic method. 


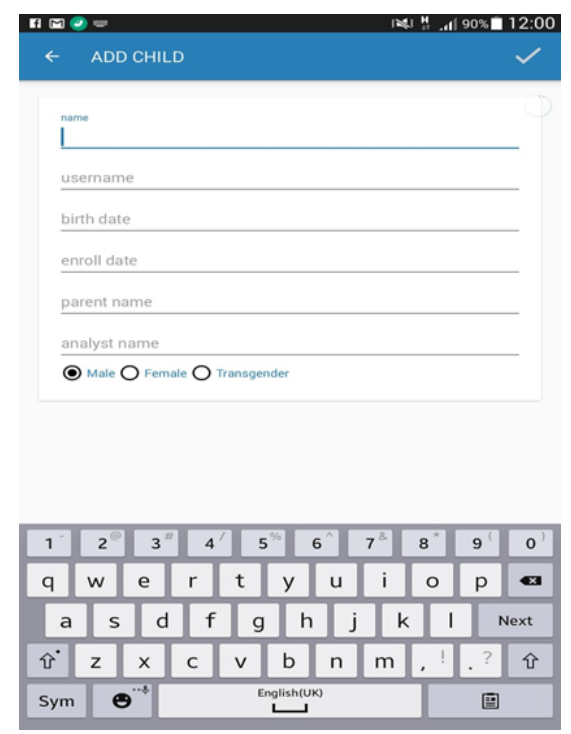

Fig. 3. Input Screen -ISAA app

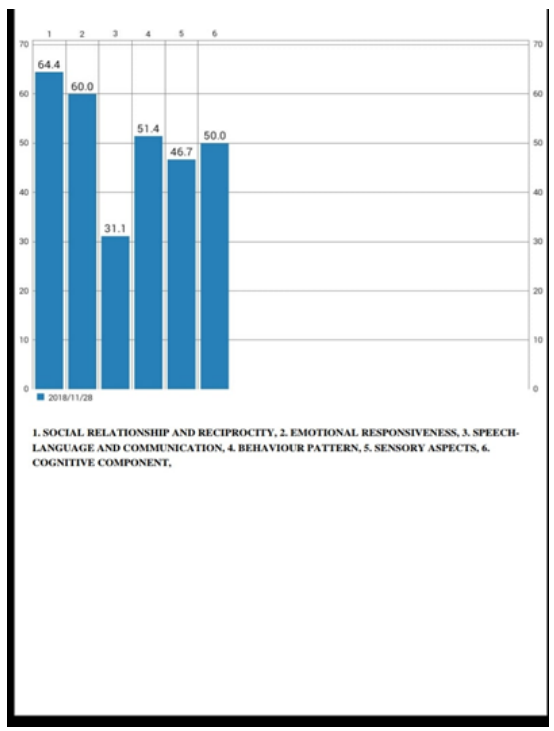

Fig. 4. Output Graph -ISAA app

\section{$5 \quad$ Acknowledgements}

We would like to express our whole-hearted appreciation to the educators and the children (Pradip Centre for Autism Management, Kolkata) participated in this study without whom this research would have never been possible.

\section{$6 \quad$ References}

$[1]<<$ Disabled persons in India a statistical profile 2016, Social Statistics Division, Govt. of India, Retrieved from http://www.mospi.gov.in

[2] Russell PS., Daniel A., Russell S., Mammen P., Abel JS., Raj LE., (2010). Diagnostic accuracy, reliability and validity of Childhood Autism Rating Scale in India. World Journal of Pediatrics. [PubMed]. https://doi.org/10.1007/s12519-010-0029-y

[3] New Delhi: Ministry of Social Justice \& Empowerment: Government of India 2009.

[4] ISAA. Report on assessment tool for autism: Indian Scale for Assessment of Autism.

[5] Indumathi Rao (2010), Portage to Early Childhood Education, JAIISH, Vol. 29(1), 128130.

[6] Reeta P., Venkatesan S. (1992), Behavioural assessment scales for Indian Children with Mental Retardation (BASIC-MR), Retrieved from http://www.niepid.nic.in/Behavioural assesment scales for indian children-basic-mr.pdf

[7] Functional Assessment Checklist for Programming, Department of Special Education, National Institute for Mentally Handicapped, Secunderabad, Retrieved from http:// www.niepid.nic.in/facp.pdf 
[8] Venkatesh, S., Phung, D., Duong, T., Greenhill, S., Adams, B. (2013), TOBY: Early intervention in autism through Technology. Proceedings of the SIGCHI, (pp. 3187 -3196). ACM. https://doi.org/10.1145/2470654.2466437

[9] Dennis W. Moore, Venkatesh S., Anderson A., Greenhill S., Phung D., Duong T., ... Whitehouse A. (2015), TOBY play-pad application to teach children with ASD -A pilot trial., Taylor \& Francis, Vol 18, 213-217. https://doi.org/10.3109/17518423.2013.784817

\section{$7 \quad$ Authors}

Arpita Mazumdar is an Assistant Professor of Dept. of Computer Science \& Engineering in Haldia Institute of Technology. She is currently pursuing her Ph. D degree under University of Engineering \& Management Kolkata. She has been awarded as WIPRO certified faculty in Java EE in 2018. Her current research interest area is machine learning and assistive technology in special education domain.

Biswajoy Chatterjee is currently Professor in Dept. of Computer Science \& Engineering in University of Engineering \& Management Kolkata. His research interest includes Nano Science \& Nano technology, assistive technology and machine learning. He is an author of $23 \mathrm{SCI}$ International Journal \& many other national journal and conferences. He is a member of Confederation of Indian Industries (CII), Computer Society of India (CSI) \& IEEE.

Mallika Banerjee is a retired Professor of University of Calcutta and now joined as Visiting Professor in IIIT, West Bengal. She is the State Awardee (Ministry of Women Development \& Social Welfare, Child Development, Health and Family Welfare), 2013, for Best Applied Research in the field of Disability. Invited and delivered Key-Note address in 'European Congress on Autism', 2019, Croatia. She has seven International and four National training on several areas of disability. Published four papers in International Journal and thirty-five papers in different esteemed peerreviewed National journals. Nineteen scholars have awarded for Doctoral thesis under supervision. Completed thirteen research projects on disability funded by UGC, ICSSR, DST, Lady Tata etc. Recent Project on 'Autism: Brain research' in collaboration with Institute of Neuroscience funded by Govt. of West Bengal.

Irfan $\mathbf{H}$ Bhati is a student of B. Tech in Information Technology in Haldia Institute of Technology. He has successfully completed various projects on IOT, Machine learning and mobile application.

Article submitted 2019-03-27. Resubmitted 2019-05-13. Final acceptance 2019-05-14. Final version published as submitted by the authors. 